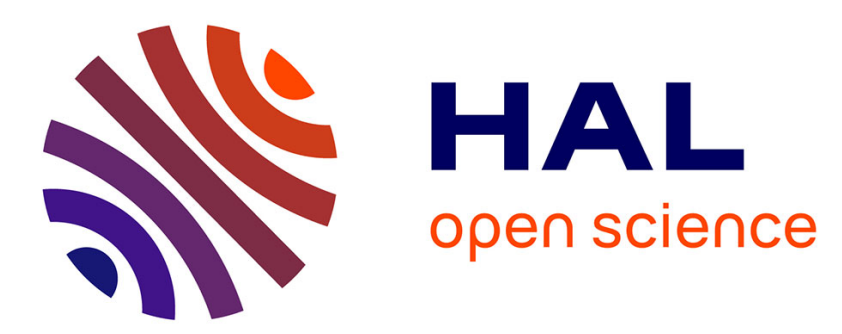

\title{
Vibrational dynamics of zero-field-splitting hamiltonian in gadolinium-based MRI contrast agents from ab initio molecular dynamics
}

Aurélie Lasoroski, Rodolphe Vuilleumier, Rodolphe Pollet

\section{To cite this version:}

Aurélie Lasoroski, Rodolphe Vuilleumier, Rodolphe Pollet. Vibrational dynamics of zero-field-splitting hamiltonian in gadolinium-based MRI contrast agents from ab initio molecular dynamics. Journal of Chemical Physics, 2014, 141, pp.014201. 10.1063/1.4885848 . hal-01157659

\author{
HAL Id: hal-01157659 \\ https://hal.science/hal-01157659
}

Submitted on 17 Nov 2015

HAL is a multi-disciplinary open access archive for the deposit and dissemination of scientific research documents, whether they are published or not. The documents may come from teaching and research institutions in France or abroad, or from public or private research centers.
L'archive ouverte pluridisciplinaire HAL, est destinée au dépôt et à la diffusion de documents scientifiques de niveau recherche, publiés ou non, émanant des établissements d'enseignement et de recherche français ou étrangers, des laboratoires publics ou privés. 


\section{AD| $\begin{aligned} & \text { The Journal of } \\ & \text { Chemical Physics }\end{aligned}$}

\section{Vibrational dynamics of zero-field-splitting hamiltonian in gadolinium-based MRI contrast agents from ab initio molecular dynamics}

Aurélie Lasoroski, Rodolphe Vuilleumier, and Rodolphe Pollet

Citation: The Journal of Chemical Physics 141, 014201 (2014); doi: 10.1063/1.4885848

View online: http://dx.doi.org/10.1063/1.4885848

View Table of Contents: http://scitation.aip.org/content/aip/journal/jcp/141/1 ?ver=pdfcov

Published by the AIP Publishing

\section{Articles you may be interested in}

Hyperfine interactions in a gadolinium-based MRI contrast agent: High-frequency modulations from ab initio simulations

J. Chem. Phys. 139, 104115 (2013); 10.1063/1.4820791

Vibrational modes of methane in the structure $\mathrm{H}$ clathrate hydrate from ab initio molecular dynamics simulation J. Chem. Phys. 137, 144306 (2012); 10.1063/1.4757914

Molecular vibrations of methane molecules in the structure I clathrate hydrate from ab initio molecular dynamics simulation

J. Chem. Phys. 136, 044508 (2012); 10.1063/1.3677231

Glycine in aqueous solution: solvation shells, interfacial water, and vibrational spectroscopy from ab initio molecular dynamics

J. Chem. Phys. 133, 114508 (2010); 10.1063/1.3481576

Ab initio simulation of a gadolinium-based magnetic resonance imaging contrast agent in aqueous solution J. Chem. Phys. 126, 181102 (2007); 10.1063/1.2736369

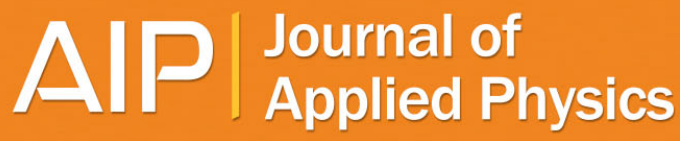

Journal of Applied Physics is pleased to announce André Anders as its new Editor-in-Chief 


\title{
Vibrational dynamics of zero-field-splitting hamiltonian in gadolinium-based MRI contrast agents from ab initio molecular dynamics
}

\author{
Aurélie Lasoroski, ${ }^{1,2}$ Rodolphe Vuilleumier, ${ }^{2,3}$ and Rodolphe Pollet ${ }^{1, a)}$ \\ ${ }^{1}$ DSM/IRAMIS/NIMBE (CEA-CNRS UMR3299), Commissariat à l'Énergie Atomique, \\ 91191 Gif-sur-Yvette, France \\ ${ }^{2}$ École Normale Supérieure, Département de Chimie, ENS-CNRS-UPMC UMR8640, 75005 Paris, France \\ ${ }^{3}$ UPMC Univ. Paris 06, 4, Place Jussieu, 75005 Paris, France
}

(Received 17 March 2014; accepted 17 June 2014; published online 3 July 2014)

\begin{abstract}
The electronic relaxation of gadolinium complexes used as MRI contrast agents was studied theoretically by following the short time evolution of zero-field-splitting parameters. The statistical analysis of $a b$ initio molecular dynamics trajectories provided a clear separation between static and transient contributions to the zero-field-splitting. For the latter, the correlation time was estimated at approximately $0.1 \mathrm{ps}$. The influence of the ligand was also probed by replacing one pendant arm of our reference macrocyclic complex by a bulkier phosphonate arm. In contrast to the transient contribution, the static zero-field-splitting was significantly influenced by this substitution. (0) 2014 AIP Publishing LLC. [http://dx.doi.org/10.1063/1.4885848]
\end{abstract}

\section{INTRODUCTION}

The influence of the electronic spin relaxation on the local $T_{1}$ shortening caused by Gd(III)-based contrast agents has been largely overlooked in medical resonance imaging (MRI) because of two other mechanisms which are much more efficient at the usual clinical fields. These are molecular tumbling and inner-sphere (IS) water exchange reaction. Together with the hydration number of the Gd(III) complex, these are the main levers that have been pulled to design better and safer paramagnetic contrast agents. In a sense, the electronic relaxation rate has already been optimized by selecting Gd among all lanthanides, which, beside its high electronic spin of $7 / 2$, exhibits a vanishing orbital angular momentum due to its $4 f^{7}$ configuration. This gives rise to an electronic relaxation time $T_{1 e}$ as long as $10^{-8} \mathrm{~s}$ at imaging fields, which enables strong hyperfine interactions between electronic and nuclear spins adjacent to $\mathrm{Gd}$, hence faster water ${ }^{1} \mathrm{H}$ relaxation.

The electronic relaxation time $T_{1 e}$ is however significantly reduced at low fields (actually below $3 \mathrm{~T}$ ) due to the modulations of the zero-field-splitting (ZFS) hamiltonian in the laboratory $(\mathrm{L})$ frame, quenching the longitudinal relaxivity of the contrast agent. In a molecular (M) frame attached to the $\mathrm{Gd}(\mathrm{III})$ complex, these modulations are actually a combination of static and transient contributions, as expressed in Ref. 1:

$$
H_{Z F S}^{(M)}(t)=H_{Z F S, s t a t}^{(M)}+H_{Z F S, t r a n s}^{(M)}(t) .
$$

While the time evolution of the first term in the (L) frame follows the slow (i.e., $>50 \mathrm{ps}$ ) reorientation of the complex, the time fluctuations of the second one reflect the fast $(<1 \mathrm{ps})$ random deformations of the coordination cage that protects the $\mathrm{Gd}^{3+}$ ion from collisions with surrounding water molecules. Electronic relaxation from this vibrational compo-

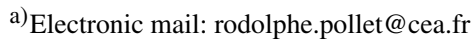

nent should thus be very small and Fries ${ }^{1}$ therefore concluded that the fluctuations of the transient ZFS will hardly influence the relaxivity, whatever the field strength, except in the case of macromolecular complexes, which exhibit very slow rotational times.

Ab initio molecular dynamics (AIMD), such as the CarParrinello method, is perfectly suited to study the interaction of $\mathrm{Gd}(\mathrm{III})$ complexes with a surrounding solvent and these sub-picosecond vibrational motions. In contrast to classical MD, the method also avoids the use of a parameterized force field by directly propagating the electronic degrees of freedom. AIMD (actually Born-Oppenheimer MD) has recently been applied to study the time evolution of various magnetic properties (including ZFS) of the $\mathrm{Ni}^{2+}$ ion in aqueous solution. ${ }^{2}$ In practice, once the AIMD trajectory has been obtained, ZFS parameters can be calculated with an electronic structure method that can be different from the one used to compute the electronic forces. This two-step approach can indeed lead to improved performances since most Car-Parrinello simulations rely on exchange-correlation density functional approximations which are only semilocal (i.e., including generalized gradient corrections). Although highlevel electron correlation methods could be used for the ZFS calculations (see, e.g., the NEVPT2 application to $\mathrm{Ni}^{2+}$ ion in water $^{3}$ ), density functional theory (DFT) is hardly avoidable given the huge number of MD configurations and the large size of the system (even if most solvent molecules can be safely eliminated). However, its applicability for the prediction of ZFS parameters has been recently questioned in the literature beyond the choice of the proper exchange-correlation density functional approximation. Depending on the authors, the computation of the spin-orbit (SO) contribution will especially vary (vide infra). Beside these unsettled issues, a more fundamental difficulty lies in the smallness of the splitting (typically less than $1 \mathrm{~cm}^{-1}$ ) to be calculated, which is due to the strong localization of the $4 f$ electrons in the core region 

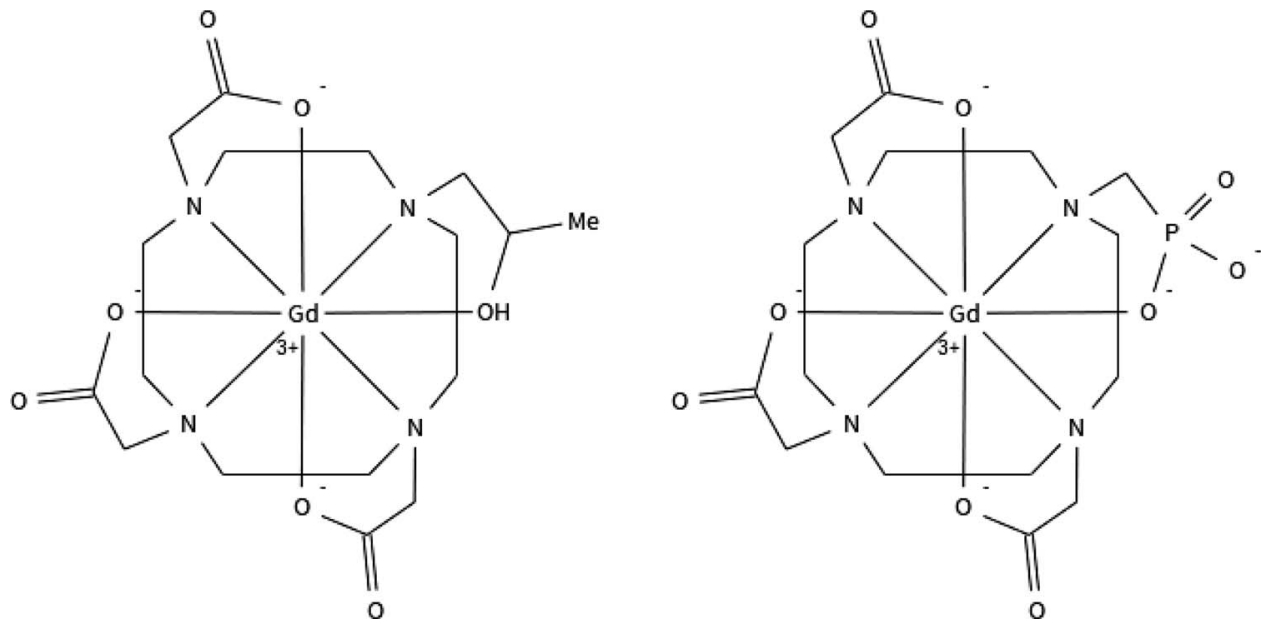

FIG. 1. Square antiprism isomers of $[\mathrm{Gd}(\mathrm{HPDO} 3 \mathrm{~A})]$ (left) and $[\mathrm{Gd}(\mathrm{DO} 3 \mathrm{AP})]^{2-}$ (right) complexes.

and to the shielding effect by the outer $5 s^{2}$ and $5 p^{6}$ closed shells. Comparisons with ESR experimental data are therefore expected to be particularly ambitious. The results reported by Kubica et al. ${ }^{4}$ for nickel(II) complexes reveal that even multiconfigurational wave function methods can sometimes fail to reproduce experimental data, while DFT calculations were judged unreliable.

In this work these questions are addressed for the specific cases of the $\left[\mathrm{Gd}(\mathrm{HPDO} 3 \mathrm{~A})\left(\mathrm{H}_{2} \mathrm{O}\right)\right]$ (or Prohance) and $\left[\mathrm{Gd}(\mathrm{DO} 3 \mathrm{AP})\left(\mathrm{H}_{2} \mathrm{O}\right)\right]^{2-}$ (a monophosphonate analogue of Dotarem) contrast agents in aqueous solution (see Figure 1).

Following our own work on the high-frequency modulations of hyperfine interactions in Prohance, ${ }^{5}$ we report here a statistical analysis of the fast ZFS fluctuations along AIMD trajectories performed in water at ambient conditions.

\section{CALCULATION OF ZFS PARAMETERS}

The ZFS spin hamiltonian acting on the $S=7 / 2$ ground state is bilinear in the electron spin operators,

$$
H_{\mathrm{ZFS}}=\mathbf{S} \cdot \mathbf{D} \cdot \mathbf{S},
$$

which can be further simplified in the principal axes of the traceless tensor $\mathbf{D}$ as follows:

$$
H_{\mathrm{ZFS}}=D\left[\hat{S}_{z}^{2}-\frac{1}{3} S(S+1)\right]+E\left(\hat{S}_{x}^{2}-\hat{S}_{y}^{2}\right)
$$

where the axial and rhombic ZFS parameters are, respectively, defined as

$$
D=D_{z z}-\frac{1}{2}\left(D_{x x}+D_{y y}\right)
$$

and

$$
E=\frac{1}{2}\left(D_{x x}-D_{y y}\right)
$$

The diagonal components of the total $D$ tensor are sorted according to the standard convention where $\left|D_{z z}\right| \geq\left|D_{y y}\right|$ $\geq\left|D_{x x}\right|$ (i.e., $0 \leq E / D \leq \frac{1}{3}$ ).

The effective hamiltonian in Eq. (2) actually incorporates direct electron spin-spin (SS) as well as SO interactions. These are first- and second-order corrections to the en- ergy, respectively. The former is a direct dipole-dipole interaction between magnetic moments of the unpaired electron spins and is usually calculated straightforwardly from the two-electron density matrix of the noninteracting Kohn-Sham (KS) system. ${ }^{6}$ In contrast and as mentioned in the Introduction, the latter has been a source of debate between several authors. Indeed, the calculation of the SO contribution from a sum-over-states formula based on Kohn-Sham spinorbitals was first proposed by Pederson and Khanna (PK), ${ }^{7}$ then modified by Neese, ${ }^{8,9}$ and eventually corrected by van Wüllen. ${ }^{10,11}$ In practice, only the prefactors of nonvanishing terms that correspond to spin-conserving $(S \rightarrow S)$ and spin-flip $(S \rightarrow S \pm 1)$ excitations will differ for each author. ${ }^{12}$ An alternative approach consists in estimating the energy differences between relativistic states using noncollinear two-component calculations, but this method requires a high numerical accuracy and is also very time-consuming. ${ }^{13}$ With this approach, results obtained for the $\mathrm{GdH}_{3}$ molecule, where $D$ is expected to be as small as $0.5 \mathrm{~cm}^{-1}$, show that local (SVWN5) and semilocal (BP86) exchange-correlation approximations provide poor estimations of $D$ in comparison to a hybrid (B3PW91) prediction, which is still two times too small. The same authors reported an exactly equivalent performance when the PK onecomponent approach was used.

\section{COMPUTATIONAL DETAILS}

\section{AIMD trajectory}

The set-up of the AIMD simulation is very similar to the one established in our study of the fast fluctuations of hyperfine tensors for the Prohance contrast agent, ${ }^{5}$ and to previous investigations of the structural and thermodynamical properties of this complex in aqueous solution. ${ }^{14,15}$ Our model system thus consists in one monohydrated Gd(III) complex in a $15.4 \AA$ cubic box including 98 supplementary water molecules (i.e., more than two hydration shells beyond the inner sphere of the complex) which satisfies periodic boundary conditions.

The time evolution of $\left[\mathrm{Gd}(\mathrm{HPDO} 3 \mathrm{~A})\left(\mathrm{H}_{2} \mathrm{O}\right)\right]$ (respectively $\left[\mathrm{Gd}(\mathrm{DO} 3 \mathrm{AP})\left(\mathrm{H}_{2} \mathrm{O}\right)\right]^{2-}$ ) was followed with a CPMD ${ }^{16}$ 
simulation of $86 \mathrm{ps}$ (respectively $18 \mathrm{ps}$ ) based on the PBE exchange-correlation functional, ${ }^{17}$ a plane wave basis set defined by a cutoff energy of $30 \mathrm{Ry}$, and ultrasoft pseudopotentials ${ }^{18}$ including scalar relativistic effects, with a nonlinear core correction ${ }^{19}$ in the case of the Gd $4 f$-in-core pseudopotential. Other parameters include a fictitious electron mass of 700 a.u., a time step of 6 a.u. (respectively 5 a.u.), and Nosé-Hoover chain thermostats (with a chain length of three) both for ions (with the target temperature set to $300 \mathrm{~K}$ ) and electrons in order to prevent heat transfer between both subsystems. To this aim, hydrogen atoms have also been replaced by deuterium atoms.

The statistical analysis of ZFS parameters along the AIMD trajectory was based on block averages. For example, in the case of Prohance, we extracted five blocks separated by 2 ps where each block included 100 configurations that were 5.8 fs apart.

\section{ZFS calculation and choice of the density functional approximation}

The computation of the ZFS parameters was performed with the ORCA program package. ${ }^{20}$ Scalar-relativistic $^{2}$ Kohn-Sham calculations (within the Zeroth Order Regular Approximation to the Dirac equation) provided unrestricted spinorbitals that were used subsequently to obtain the second-order SO contribution. For the first-order SS term, unrestricted natural orbitals (UNOs) were used instead, as recommended in the literature. ${ }^{21}$ Our benchmark calculations (see the supplementary material ${ }^{22}$ ) on the $\mathrm{Gd}(\mathrm{III})$ aquo ion and the $\left[\mathrm{Gd}(\mathrm{HPDO} 3 \mathrm{~A})\left(\mathrm{H}_{2} \mathrm{O}\right)\right]$ complex show that the all-electron, uncontracted, double and triple zeta basis sets provide almost identical axial and rhombic ZFS values so that the more economical polarized splitvalence (SVP) basis set was eventually selected. As for the exchange-correlation density functional approximation, similar results were obtained with GGAs (BLYP and PBE) and one meta-GGA (TPSS) whereas hybrid approximations (B3LYP and PBE0) predicted much larger splittings $\left(>1 \mathrm{~cm}^{-1}\right)$, which is quite unexpected given the high symmetry of the $\mathrm{Gd}(\mathrm{III})$ electronic configuration. For example, the experimental axial ZFS parameter of the $\left[\mathrm{Gd}(\mathrm{DOTA})\left(\mathrm{H}_{2} \mathrm{O}\right)\right]^{-}$ complex obtained from EPR spectra in frozen solution by the Lausanne group ${ }^{23}$ is $-0.019 \mathrm{~cm}^{-1}$. On closer inspection, the presence of a fractional amount of parallel-spin correlation (i.e., exact exchange) in hybrid approximations caused a strong stabilization (respectively destabilization) of occupied $4 f_{\alpha}$ (respectively empty $4 f_{\beta}$ ) spinorbitals, resulting in a $4 f-$ $4 f$ separation more than twice as large as GGAs or meta-GGA (see Table I). Instead of lying between the valence and the conduction bands, the $4 f_{\beta}$ spinorbitals were even pushed up into the conduction band. In addition, as expected from hybrid approximations, the size of the KS band gap also increased (see Table I). These changes in the electronic structure of the complex directly affected the second-order SO contribution (where differences between spinorbital energies appear in denominators), especially the $\alpha \rightarrow \beta$ (spin-flip) term, significantly overestimating the total ZFS splitting (since the SS contribution remains very small for
TABLE I. Decomposition of the SO contribution to axial ZFS splitting $D_{\sigma \sigma^{\prime}}^{S O}$ in terms of excitations (in $\mathrm{cm}^{-1}$ ) for a representative geometry of the $\left[\mathrm{Gd}(\mathrm{HPDO} 3 \mathrm{~A})\left(\mathrm{H}_{2} \mathrm{O}\right)\right]$ complex with two additional second-sphere water molecules. The cases of GGA (PBE), meta-GGA (TPSS), and hybrid (PBE0) approximations are compared by also reporting their respective $\mathrm{KS}$ band gaps (i.e., between valence and conduction bands), valence to $4 f_{\beta}$ and $4 f-4 f$ separations. The PK method, ZORA approximation, and decontracted TZVPP basis set were used.

\begin{tabular}{lccc}
\hline \hline$D_{\sigma \sigma^{\prime}}^{S O}$ & PBE & TPSS & PBE0 \\
\hline$\alpha \alpha$ & -0.990 & -1.009 & -1.015 \\
$\beta \beta$ & -1.187 & -1.138 & -1.002 \\
$\alpha \beta$ & 0.998 & 1.013 & -2.473 \\
$\beta \alpha$ & 0.888 & 0.938 & -0.281 \\
Total & -0.291 & -0.196 & -4.771 \\
KS band gap $(\mathrm{eV})$ & 3.1 & 3.4 & 5.4 \\
Valence to $4 f_{\beta}(\mathrm{eV})$ & 2.0 & 2.5 & 7.3 \\
$4 f-4 f$ separation $(\mathrm{eV})$ & 5.0 & 5.1 & 12.6 \\
\hline \hline
\end{tabular}

all approximations). For these reasons, TPSS was eventually preferred to B3LYP or PBE0 approximations.

\section{RESULTS AND DISCUSSIONS}

\section{Static ZFS of [Gd(HPDO3A)( $\left.\left.\mathrm{H}_{2} \mathrm{O}\right)\right]$}

Given the short length (less than 100 ps) of our AIMD trajectory, no rotation of the complex was observed therefore (L) and (M) frames can be considered identical. The static parameters of the ZFS can then be obtained after diagonalization of the time average of the total ZFS tensor in the (L) frame (see Figure 2 and Table II).

First we note that both methods predict a small rhombicity. The sign of $D$ is also always negative, corresponding to

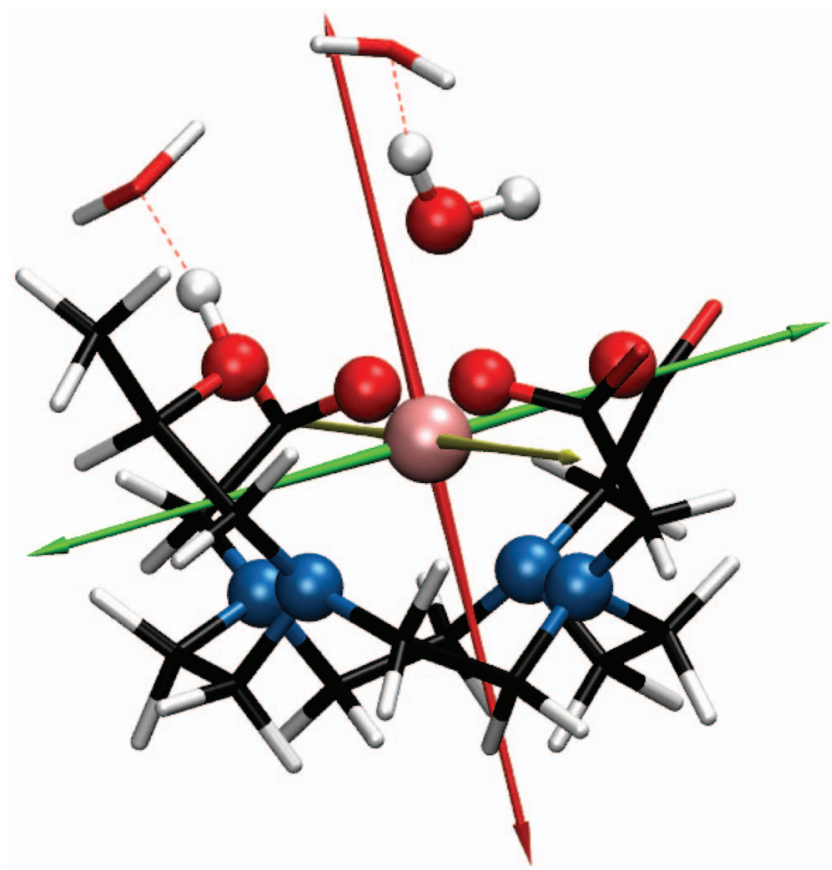

FIG. 2. Snapshot of the AIMD trajectory of $\left[\mathrm{Gd}(\mathrm{HPDO} 3 \mathrm{~A})\left(\mathrm{H}_{2} \mathrm{O}\right)\right]$ in aqueous solution (showing only one IS and two SS water molecules) together with the corresponding eigenvectors of the total ZFS tensor. 
TABLE II. Static ZFS parameters in $\mathrm{cm}^{-1}$ of $\left[\mathrm{Gd}(\mathrm{HPDO} 3 \mathrm{~A})\left(\mathrm{H}_{2} \mathrm{O}\right)\right]$ according to Pederson-Khanna (PK) and Neese methods (vide supra for references and for details on block averages).

\begin{tabular}{lccc}
\hline \hline Method & $D$ & $E$ & $E / D$ \\
\hline PK & -0.200 & -0.0186 & 0.0929 \\
Neese & -0.296 & -0.0257 & 0.0868 \\
\hline \hline
\end{tabular}
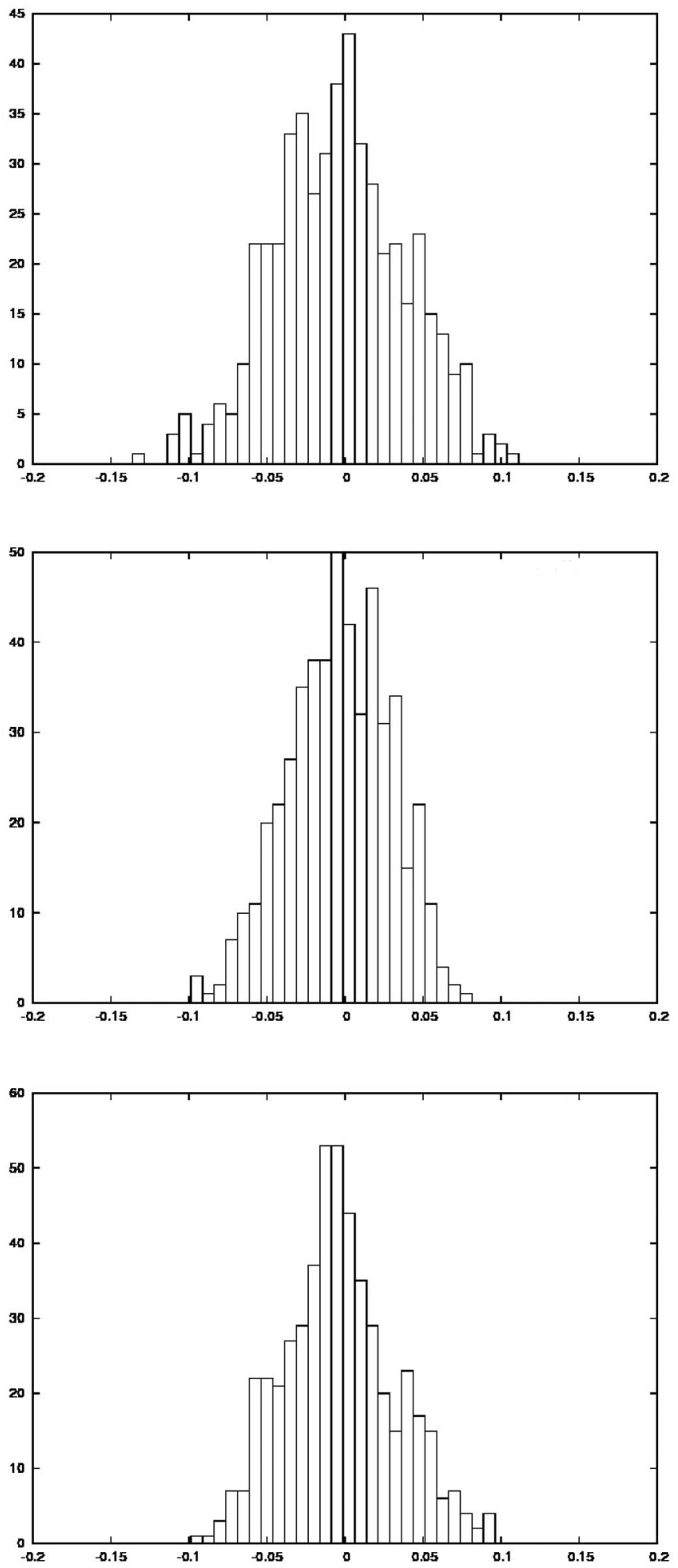

a ground state with $m_{S}= \pm 7 / 2$. This is in agreement with the analysis of EPR spectra in water-glycerol glass for macrocyclic compounds by Benmelouka et $a l .{ }^{23}$ From the axial and rhombic parameters, the magnitude of static ZFS can then be calculated according to $\Delta=\sqrt{\frac{2}{3} D^{2}+2 E^{2}}$. It amounts to 3.12 and $4.60 \times 10^{10} \mathrm{rad} \mathrm{s}^{-1}$ with the PK and Neese methods, respectively. A recent ${ }^{1} \mathrm{H}$ and ${ }^{17} \mathrm{O}$ relaxometric study
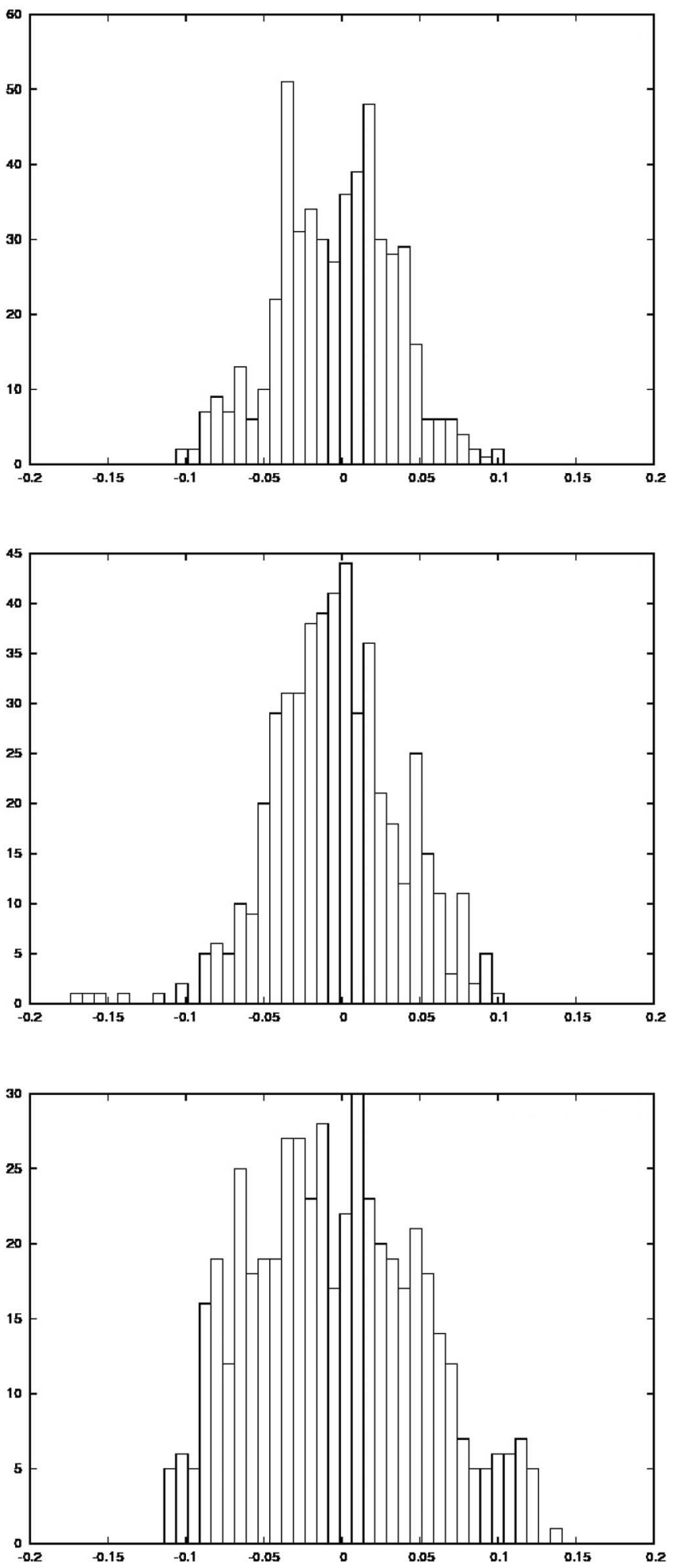

FIG. 3. From top left to bottom right: distributions of the $d_{11}, d_{12}, d_{13}, d_{22}, d_{23}$, and $d_{33}$ coefficients (in $\mathrm{cm}^{-1}$ ) along the AIMD trajectory of $\left[\mathrm{Gd}(\mathrm{HPDO} 3 \mathrm{~A})\left(\mathrm{H}_{2} \mathrm{O}\right)\right]$ (with Neese method for SO contribution, see text). 
of Prohance ${ }^{24}$ reports a ZFS magnitude for the square antiprism isomer of Prohance equal to $0.99 \times 10^{10} \mathrm{rad} \mathrm{s}^{-1}$. Although the latter experimental value does not separate static from transient contributions, it seems that our theoretical ap- proach gives the correct order of magnitude of the static ZFS but still overestimates it. The same conclusion was drawn by Senn et al., ${ }^{25}$ who used ligand field density functional theory (LF-DFT) with LDA, GGA, or hybrid approximations to
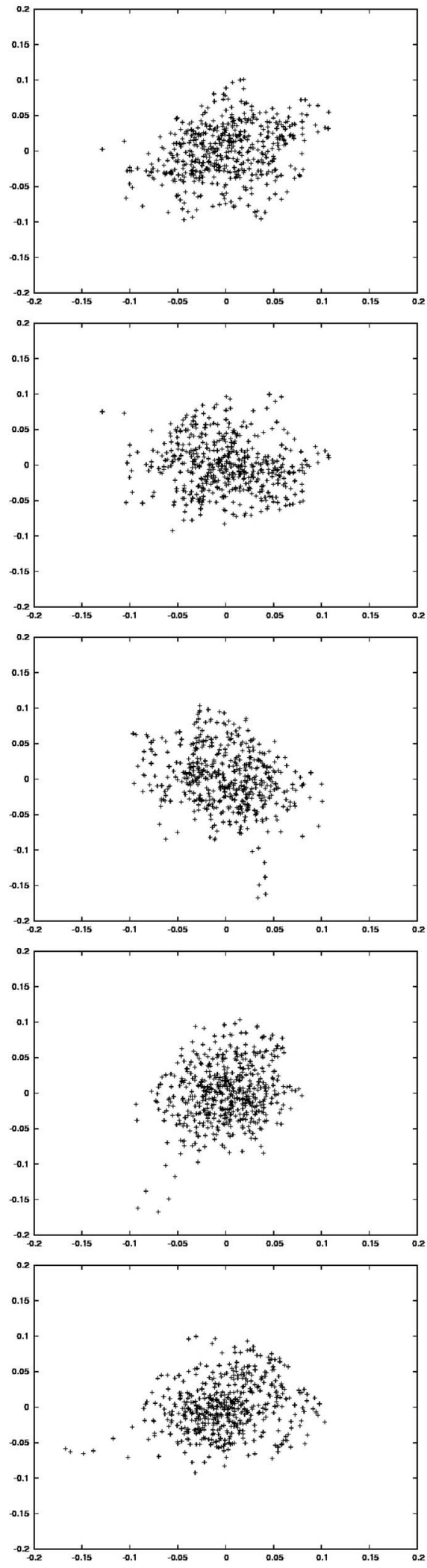
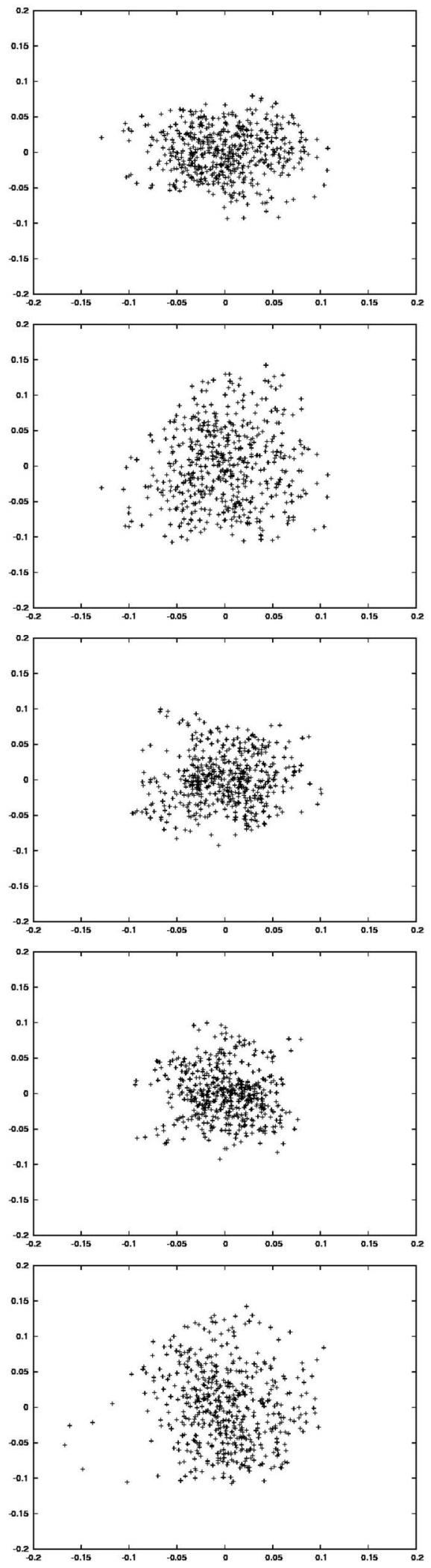
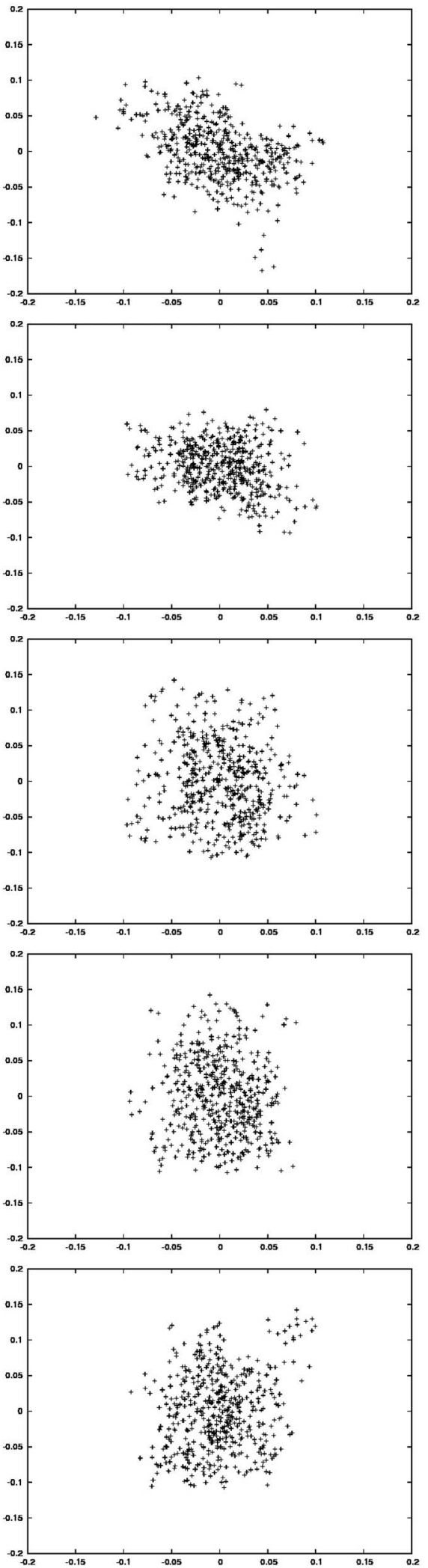

FIG. 4. From top left to bottom right: dependence between $d_{11}$ and $d_{12}, d_{11}$ and $d_{13}, d_{11}$ and $d_{22}, d_{11}$ and $d_{23}, d_{11}$ and $d_{33}, d_{12}$ and $d_{13}, d_{12}$ and $d_{22}, d_{12}$ and $d_{23}, d_{12}$ and $d_{33}, d_{13}$ and $d_{22}, d_{13}$ and $d_{23}, d_{13}$ and $d_{33}, d_{22}$ and $d_{23}, d_{22}$ and $d_{33}, d_{23}$ and $d_{33}$ coefficients (in $\mathrm{cm}^{-1}$ ) along the AIMD trajectory of $\left[\mathrm{Gd}(\mathrm{HPDO} 3 \mathrm{~A})\left(\mathrm{H}_{2} \mathrm{O}\right)\right]$ (with Neese method for SO contribution, see text). 
study the $\left[\mathrm{Gd}(\mathrm{DOTA})\left(\mathrm{H}_{2} \mathrm{O}\right)\right]^{-}$complex. Their calculated ZFS magnitudes were however more than one order of magnitude as large as the experimental reference. In addition, LF-DFT predicted the wrong sign for $D$, hence a ground state with $m_{S}$ $= \pm 1 / 2$.

\section{Transient ZFS of [Gd(HPDO3A)( $\left.\mathrm{H}_{2} \mathrm{O}\right)$ ]}

By substracting the static contribution to every instantaneous ZFS tensors (see Eq. (1)), the transient tensors can then be obtained. These are symmetric $3 \times 3$ matrices,

$$
\mathcal{D}_{\text {ZFS, trans }}=\left(\begin{array}{lll}
d_{11} & d_{12} & d_{13} \\
d_{12} & d_{22} & d_{23} \\
d_{13} & d_{23} & d_{33}
\end{array}\right),
$$

whose coefficients $d_{i j}$ satisfy a nearly gaussian distribution (see Fig. 3). They are mostly uncorrelated, as can be seen in Fig. 4. The largest Pearson's correlation coefficient indeed amounts to -0.4 , for the $\left(d_{11}, d_{22}\right)$ pair, revealing a weak linear relationship between them.

In order to characterize the time fluctuations of the transient contribution, the average of the normalized time autocorrelation functions of all nine coefficients has been calculated,

$$
C(t)=\frac{1}{9} \sum_{i, j=1}^{3} \frac{\left\langle d_{i j}(0) d_{i j}(t)\right\rangle}{\left\langle d_{i j}(0) d_{i j}(0)\right\rangle} .
$$

Figure 5 shows that $C(t)$ is smoother when the Neese method is used. In both cases, a fast initial decay followed by anticorrelation is observed. Fitting these data to a monoexponential function, $\exp \left(\frac{-t}{\tau_{c}}\right) \cos (2 \pi c v t)$, provides a correlation time $\tau_{c}$ of $95.3 \mathrm{fs}$ (respectively $53.4 \mathrm{fs}$ ) and a frequency $v$ of $171.82 \mathrm{~cm}^{-1}$ (respectively $162.35 \mathrm{~cm}^{-1}$ ) with Neese (respectively PK) method. Therefore we can infer that the correlation time of the transient contribution to the ZFS tensor is close to $0.1 \mathrm{ps}$. This results confirms what Odelius et al. ${ }^{26}$ reported almost 20 years ago using classical MD and a preparameter-

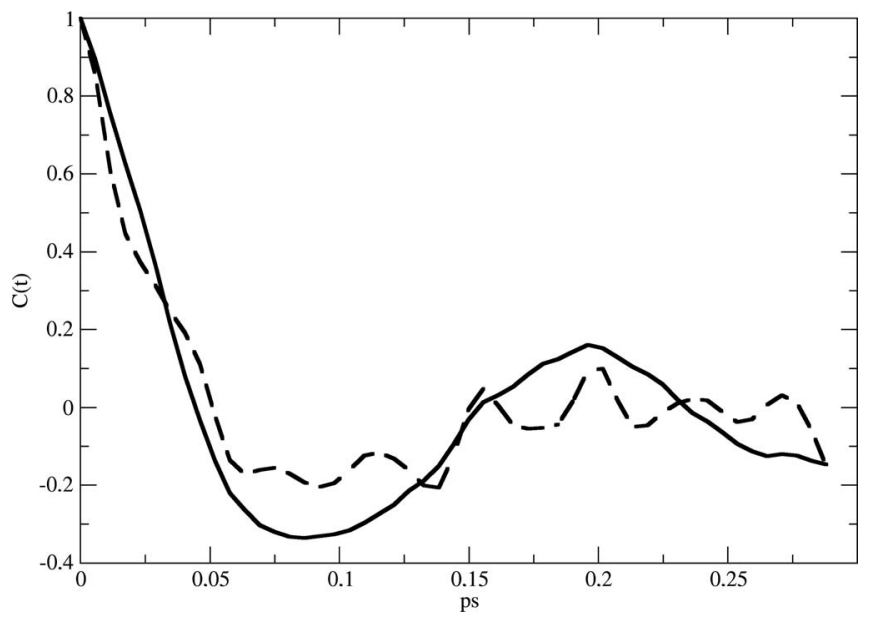

FIG. 5. Normalized time autocorrelation functions of the transient contribution (see text) for [ $\left.\mathrm{Gd}(\mathrm{HPDO} 3 \mathrm{~A})\left(\mathrm{H}_{2} \mathrm{O}\right)\right]$, obtained with the Neese (solid line) and PK (dashed line). ized ZFS hypersurface for $\mathrm{Ni}(\mathrm{II})$ in aqueous solution. More recently, the simulation of the $\mathrm{Ni}^{2+}$ ion in water was revisited by other authors using, instead, AIMD simulation. ${ }^{2}$ In contrast to Odelius et al. and to our own work, the time autocorrelation function of the ZFS tensor was so damped that no oscillation was observed. Another theoretical study, again based on classical MD, assumed that the dynamics of the electric field gradient at the metal ion generated by the surrounding water molecules should be qualitatively the same as for the transient ZFS interaction. ${ }^{27}$ Using this indirect approach, the authors also concluded in the existence of damped oscillations at short times for the $\mathrm{Gd}^{3+}$ ion in water. Their correlation time of 125 fs agrees fairly well with our estimation; they obtain a much higher frequency of $414 \mathrm{~cm}^{-1}$. Fitting the correlation function generated by the pseudorotation mode ${ }^{28}$ with a multiexponential function, they also found a longer, intermediate, time constant of 1-2 ps. Still, the integrated correlation time was below the picosecond. Due to the high computational cost of our $a b$ initio approach, we could not observe this intermediate component.

The short correlation time that we observe in our simulation contrasts with much longer correlation times found from NMRD or EPR experiments, of the order of several picoseconds ${ }^{29-33}$ and a characteristic time of 8 ps has been reported for $[\mathrm{Gd}(\mathrm{HPDO} 3 \mathrm{~A})(\mathrm{H} 2 \mathrm{O})] .{ }^{24}$ From these experiments however, the nature of the molecular motion leading to the observed decorrelation cannot be determined. Fries and Belorizky ${ }^{34}$ have pointed out that the transient ZFS is often described using the pseudo-rotation model and the static ZFS often neglected, which leads to possible observation of an effective correlation time intermediate between the vibrational and rotational characteristic times. With a proper separation of vibrations and rotations, these authors suggest that the correlation time for the transient ZFS would be found between $0.1 \mathrm{ps}$ and $1 \mathrm{ps}$. Simulations suggest a very short timescale for the decorrelation arising from fast vibrations or non-activated deformations of the molecular structure. These fluctuations are so fast that their signature may be analogous to a decrease of the amplitude of the ZFS. This then hints to a different molecular origin of the correlation times of several picoseconds among which hydrogen bond rearrangement or largeamplitude activated deformations.

\section{Influence of the ligand}

We now compare the previous results with analogous calculations for the $\left[\mathrm{Gd}(\mathrm{DO} 3 \mathrm{AP})\left(\mathrm{H}_{2} \mathrm{O}\right)\right]^{2-}$ complex synthesized by Rudovsky et al. ${ }^{35}$ The chelate is not neutral anymore but bears a negative charge. In addition, the presence of a bulky phosphonate pendant arm increases the asymmetry of the coordination cage. A structural analysis of our AIMD trajectory reveals that, in comparison with Prohance, the distance between $\mathrm{Gd}$ and the IS oxygen $\left(\mathrm{O}^{I S}\right)$ increases from $2.55 \AA$ to $2.67 \AA$, the dipole of the IS water is less aligned with the $\mathrm{Gd}-\mathrm{O}^{I S}$ vector, and the number of hydrogen bonds between $\mathrm{O}^{I S}$ and other water molecules is much larger. In short, the slightly farther IS water is more exposed to solvent. 
TABLE III. Static ZFS parameters in $\mathrm{cm}^{-1}$ of $\left[\mathrm{Gd}(\mathrm{DO} 3 \mathrm{AP})\left(\mathrm{H}_{2} \mathrm{O}\right)\right]^{2-}$ according to Pederson-Khanna (PK) and Neese methods (vide supra for references and for details on block averages).

\begin{tabular}{lccc}
\hline \hline Method & $D$ & $E$ & $E / D$ \\
\hline PK & -0.285 & -0.0488 & 0.171 \\
Neese & -0.418 & -0.0582 & 0.139 \\
\hline \hline
\end{tabular}

The static ZFS parameters of $\left[\mathrm{Gd}(\mathrm{DO} 3 \mathrm{AP})\left(\mathrm{H}_{2} \mathrm{O}\right)\right]^{2-}$ are reported in Table III. Both axial and rhombic contributions have increased in comparison with the Prohance case. The parameter $E$ is especially 2-3 times larger, which was expected given the higher asymmetry of the complex.

In contrast, the time evolution of the transient contribution is rather similar to the case of Prohance, with a correlation time $\tau_{c}$ of $89.5 \mathrm{fs}$ and a frequency $v$ of $124.15 \mathrm{~cm}^{-1}$. This is in agreement with the EPR study of acyclic, macrocyclic, phosphinate, and cryptand complexes by Benmelouka et al. ${ }^{36}$ who concluded that "whereas the static ZFS clearly depends on the nature of the chelating ligand, the transient ZFS does not."

\section{CONCLUSION}

In our attempt to study the vibrational dynamics of zero-field-splitting with AIMD simulations, we first noticed that, for Gd(III) complexes, hybrid approximations to the exchange-correlation functional significantly overestimate the magnitude of the splitting. We made the assumption that this shortcoming was related to the positions of the $4 f$ (empty and occupied) orbitals in the energy spectrum. GGA's and metaGGA's approximations therefore provided a better estimation of the spin-orbit contribution to ZFS.

Even with the most accurate density functional (here TPSS), we observed that our theoretical approach tends to overestimate the magnitude of the static ZFS with respect to experimental data. However, in contrast to other theoretical methods, we could predict the correct sign for the axial ZFS parameter, which corresponds to a ground state with $m_{S}= \pm 7 / 2$.

The transient ZFS tensors were obtained after the subtraction of this static contribution to each instantaneous ZFS tensor. Since our calculations show that their coefficients satisfy a nearly gaussian distribution and lose correlation after approximately $0.1 \mathrm{ps}$, we suggest that the dynamics of transient ZFS could be safely modeled by a gaussian process.

We also probed the influence of the ligand by replacing one of the pending arm of the Prohance contrast agent by a bulkier phosphonate arm. For this non-neutral complex, the static ZFS (especially rhombic) parameters were much larger while the correlation time corresponding to the transient contribution was almost unchanged.
We expect that, in the near future, new algorithms and technologies will push the frontiers of AIMD in order to reach longer timescales. This is indeed required if the whole dynamics of static ZFS (which should include the slow molecular tumbling) is to be studied theoretically.

${ }^{1}$ P. H. Fries, Eur. J. Inorg. Chem. 2012, 2156 (2012).

${ }^{2}$ J. Mares, H. Liimatainen, T. O. Pennanen, and J. Vaara, J. Chem. Theory Comput. 7, 3248 (2011).

${ }^{3}$ J. Mares, M. Hanni, P. Lantto, J. Lounila, and J. Vaara, Phys. Chem. Chem. Phys. 16, 6916 (2014).

${ }^{4}$ A. Kubica, J. Kowalewski, D. Kruk, and M. Odelius, J. Chem. Phys. 138, 064304 (2013).

${ }^{5}$ A. Lasoroski, R. Vuilleumier, and R. Pollet, J. Chem. Phys. 139, 104115 (2013).

${ }^{6}$ T. T. Petrenko, T. L. Petrenko, and V. Y. J. Bratus, J. Phys. Condens. Matter 14, 12433 (2002).

${ }^{7}$ M. R. Pederson and S. N. Khanna, Phys. Rev. B 60, 9566 (1999).

${ }^{8}$ F. Neese, J. Am. Chem. Soc. 128, 10213 (2006).

${ }^{9}$ F. Neese, J. Chem. Phys. 127, 164112 (2007).

${ }^{10}$ C. van Wüllen, J. Phys. Chem. A 113, 11535 (2009)

${ }^{11}$ C. van Wüllen, J. Chem. Phys. 130, 194109 (2009).

${ }^{12}$ S. Schmitt, P. Jost, and C. van Wüllen, J. Chem. Phys. 134, 194113 (2011)

${ }^{13}$ R. Reviakine, A. V. Arbuznikov, J.-C. Tremblay, C. Remenyi, O. L. Malkina, V. G. Malkin, and M. Kaupp, J. Chem. Phys. 125, 054110 (2006).

${ }^{14}$ R. Pollet and D. Marx, J. Chem. Phys. 126, 181102 (2007).

${ }^{15}$ R. Pollet, N. Nair, and D. Marx, Inorg. Chem. 50, 4791 (2011).

${ }^{16} \mathrm{~J}$. Hutter et al., CPMD Software Package, see www.cpmd.org.

${ }^{17}$ J. P. Perdew, K. Burke, and M. Ernzerhof, Phys. Rev. Lett. 77, 3865 (1996); 78, 1396 (1997) (Erratum)

${ }^{18}$ D. Vanderbilt, Phys. Rev. B 41, 7892 (1990).

${ }^{19}$ D. Marx and J. Hutter, Ab initio Molecular Dynamics: Basic Theory and Advanced Methods (Cambridge University Press, Cambridge, 2009).

${ }^{20}$ F. Neese, Comput. Mol. Sci. 2, 73 (2012).

${ }^{21}$ S. Sinnecker and F. Neese, J. Phys. Chem. A 110, 12267 (2006).

${ }^{22}$ See supplementary material at http://dx.doi.org/10.1063/1.4885848 for benchmarks related to the choice of the density functional, the spin-orbit method, and the basis set.

${ }^{23}$ M. Benmelouka, J. van Tol, A. Borel, M. Port, L. Helm, L. C. Brunel, and A. E. Merbach, J. Am. Chem. Soc. 128, 7807 (2006).

${ }^{24}$ D. D. Castelli, M. C. Caligara, M. Botta, E. Terreno, and S. Aime, Inorg. Chem. 52, 7130 (2013).

${ }^{25}$ F. Senn, L. Helm, A. Borel, and C. A. Daul, C. R. Chim. 15, 250 (2012).

${ }^{26}$ M. Odelius, C. Ribbing, and J. Kowalewski, J. Chem. Phys. 103, 1800 (1995).

${ }^{27}$ M. Lindgren, A. Laaksonen, and P.-O. Westlund, Phys. Chem. Chem. Phys. 11, 10368 (2009).

${ }^{28}$ P.-O. Westlund, N. Benetis, and H. Wennerstrom, Mol. Phys. 61, 177 (1987).

${ }^{29}$ D. H. Powell, O. M. N. Dhubhghaill, D. Pubanz, L. Helm, Y. S. Lebedev, W. Schlaepfer, and A. E. Merbach, J. Am. Chem. Soc. 118, 9333 (1996).

${ }^{30}$ P. Caravan, J. J. Ellison, T. J. McMurry, and R. B. Lauffer, Chem. Rev. 99, 2293 (1999).

${ }^{31}$ D. Kruk, T. Nilsson, and J. Kowalewski, Phys. Chem. Chem. Phys. 3, 4907 (2001).

${ }^{32}$ L. Tei, M. Botta, C. Lovazzano, A. Barge, L. Milone, and S. Aime, Magn. Reson. Chem. 46, S86 (2008).

${ }^{33}$ D. J. Mastarone, V. S. R. Harrison, A. L. Eckermann, G. Parigi, C. Luchinat, and T. J. Meade, J. Am. Chem. Soc. 133, 5329 (2011).

${ }^{34}$ P.-H. Fries and E. Belorizky, ChemPhysChem 13, 2074 (2012).

${ }^{35}$ J. Rudovsky, P. Cigler, J. Kotek, P. Hermann, P. Vojtisek, I. Lukes, J. A. Peters, L. V. Elst, and R. N. Muller, Chem. Eur. J. 11, 2373 (2005).

${ }^{36}$ M. Benmelouka, A. Borel, L. M. L. Helm, and A. E. Merbach, J. Phys. Chem. B 111, 832 (2007). 